\title{
A New Nonrelativistic Atomic Energy Spectrum of Energy Dependent Potential for Heavy Quarkouniom in Noncommutative Spaces and Phases Symmetries
}

\author{
Abdelmadjid Maireche* \\ Laboratory of Physics and Material Chemistry, Physics department, Sciences Faculty, \\ University of M'sila-M'sila, Algeria
}

(Received 18 February 2016; revised manuscript received 10 June 2016; published online 21 June 2016)

The main objective of this search work is to study a three dimensional space-phase modified Schrödinger equation with energy dependent potential plus three terms: $\frac{g}{r^{4}},-\frac{1}{2} \mu \omega^{2}\left(1+\gamma E_{n, l}\right)$ and $\frac{\overrightarrow{\mathbf{L}} \overrightarrow{\vec{\theta}}}{2 \mu}$ is carried out. Together with the Boopp's shift method and standard perturbation theory the new energy spectra shown to be dependent with new atomic quantum in the non-commutative three dimensional real spaces and phases symmetries (NC-3D: RSP) and we have also constructed the corresponding deformed noncommutative Hamiltonian for studied potential.

Keywords: Energy depended potential, quarkouniom systems, noncommutative space, noncommutative phase and Boopp's shift method.

DOI: 10.21272/jnep.8(2).02046

PACS numbers: 11.10.Nx, 32.30. - r, 03.65. - w

\section{INTRODUCTION}

The search for exact bound-state solutions of nonrelativistic Schrödinger equation for central and noncentral potentials have been a subject of interest in materiel sciences, quantum mechanics and different fields of sciences [1-25]. In recently years, the Schrödinger equation were extended to noncommutative space and phase to gives profound physical and chemical interpretations of different fields at Nano and Plank's scales [26-51]. In particularly, the energy spectrum of heavy quarkouniom $(c \bar{c}$ and $b \bar{b})$ is rich source of information on the nature of the interquark force at distance inferior $0.1 \mathrm{fm}$ and superior to $1.0 \mathrm{fm}$ [23], we want to extend this work to noncommutativity of space and phase to discover the new symmetries. The idea of noncommutativity was introduced by H. Snyder [51]. Recently the new structure of space and phase has been developed by formalism of star product, Boopp's shift method and the Seiberg-Witten map, the new product, known by star product between two arbitrary functions $f(x)$ and $g(x)$ (noted by $f(x) * g(x))$ in first order of two parameters $\theta^{\mu \nu}$ and $\bar{\theta}^{\mu \nu}$ [26-50]:

$$
\begin{aligned}
& f(x) * g(x)=f(x) g(x)-\frac{i}{2} \theta^{\mu v} \partial_{\mu}^{x} f(x) \partial_{\nu}^{x} g(x)- \\
& -\frac{i}{2} \bar{\theta}^{\mu v} \partial_{\mu}^{p} f(x) \partial_{v}^{p} g(x)
\end{aligned}
$$

The parameters $\theta^{\mu v}$ and $\bar{\theta}^{\mu v}$ are an antisymmetric real matrix; the above star-product allow us to obtain the two new commutators:

$$
\left[\hat{x}_{\mu}, \hat{x}_{v}\right]_{*}=i \theta_{i j} \text { and }\left[\hat{p}_{\mu}, \hat{p}_{v}\right]_{*}=i \bar{\theta}_{i j}
$$

In present work, we apply Boopp's shift method in- stead of solving the deformed Schrödinger equation in noncommutative three dimensional real space phase (NC-3D:RSP) with star product, the Schrödinger equation will be treated directly by using the two commutators, in addition to usual commutator on quantum mechanics [30-50]:

$$
\left[\hat{x}_{i}, \hat{x}_{j}\right]=i \theta_{i j} \text { and }\left[\hat{p}_{i}, \hat{p}_{j}\right]=i \bar{\theta}_{i j}
$$

the new two operators $\hat{x}_{i}$ and $\hat{p}_{i}$ are depended with ordinary operator $x_{i}$ and $p_{i}$ from projections relations, respectively $(c=\hbar=1)$ :

$$
\hat{x}_{i}=x_{i}-\frac{\theta_{i j}}{2} p_{j} \text { And } \hat{p}_{i}=p_{i}-\frac{\bar{\theta}_{i j}}{2} x_{j}
$$

The rest of present paper is organized as follows: in the next section, we present briefly review of energy spectrum of heavy quarkouniom in ordinary three dimensional spaces. Section 3 is devoted to present Boopp's shift method formalism and determine the deformed energy dependent potential in (NC-3D: RSP). Section 4 is devoted to derive the noncommutative spin-orbital Hamiltonian operator for modified energy dependent potential in (NC_3D: RSP), the exact spin-orbital spectrum for spinorbital Hamiltonian and the exact magnetic spectrum for spin-orbital Hamiltonian. In section 5, we resume the global spectrum for energy dependent potential and the total noncommutative Hamiltonian operator. Some concluding results are given in last section.

\section{BRIEFLY REVIEW OF ENERGY SPECTRUM OF HEAVY QUARKOUNIOM IN ORDINARY THREE DIMENSIONAL SPACES}

In this section, we shall gives a briefly review of energy spectrum of heavy quarkouniom systems

\footnotetext{
*abmaireche@gmail.com
} 
$(c \bar{c}$ and $b \bar{b})$ in framework of non-relativistic Schrödinger equation using interquark potential [23]:

$$
V\left(r, E_{n, l}\right)=\frac{1}{2} \mu \omega^{2} r^{2}\left(1+\gamma E_{n, l}\right)+\frac{g}{r^{2}}
$$

where $\omega, g$ and $\gamma$ are constants. The three dimensional Schrödinger equation is [23]:

$$
\begin{aligned}
& \left(-\frac{\Delta}{2 \mu}+\frac{1}{2} m \omega^{2} r^{2}\left(1+\gamma E_{n, l}\right)+\frac{g}{r^{2}}\right) \Psi(r, \theta, \varphi)= \\
& =E_{n, l} \Psi(r, \theta, \varphi)
\end{aligned}
$$

The reduced mass $\mu$ in terms quark mass $m_{q}$ and antiquark mass $m_{q}^{-}$:

$$
\mu=\left\{\begin{array}{l}
\frac{m_{c} m_{\bar{c}}}{m_{c}+m_{\bar{c}}} \text { for } \quad \bar{c} \\
\frac{m_{b} m_{\bar{b}}}{m_{b}+m_{\bar{b}}} \text { for } \quad b \bar{b}
\end{array} .\right.
$$

The method of separation of variable has been applied in two references [23, 24]:

$$
\Psi_{n, l, m}(\vec{r})=\frac{u_{n, l}(r)}{r} Y_{l, m}(\theta, \varphi)
$$

Then, eq. (7) reduces to [23, 24]:

$$
t \frac{d^{2} u_{n, l}(t)}{d t^{2}}+\frac{d u_{n, l}(t)}{d t}+\frac{1}{4}\left[\alpha-\frac{\beta}{t}-t\right] u_{n, l}(t)=0
$$

where $t=m \omega^{\prime} r^{2}, \alpha=\frac{2 E}{\omega^{\prime}}, \beta=l(l+1)+2 \mu g$ and $\omega^{\prime 2}=\omega^{2}(1+\gamma E)$. Eq. (8) accepts a solution for the form $[23,24]$ :

$$
u_{n, l}(t)=t^{\eta} \exp \left(-\frac{t}{2}\right) R_{l}(t)
$$

where $\eta=\frac{1}{4}\left(1+\sqrt{(2 l+1)^{2}+8 \mu g}\right)$ and $R_{l}(t)$ satisfies the following equation $[23,24]$ :

$t \frac{d^{2} R_{l}(t)}{d t^{2}}+\left[2 \eta+\frac{1}{2}-t\right] \frac{d R_{l}(t)}{d t}-\left[\eta+\frac{1}{4}-\frac{\alpha}{4}\right] R_{l}(t)=0$ (10)

The non-singular solution of the above equation is the confluent hypergeometric series [23, 24]:

$$
R_{l}(t)=F\left(\eta+\frac{1}{4}-\frac{\alpha}{4}=n_{r}, 2 \eta+\frac{1}{2} ; t\right)
$$

The normalized eignenfunctions and the discrete energy eigenvalue of the energy dependent potential are as given by $[23,24]$ :

$$
\begin{gathered}
\Psi_{n, l, m}(\vec{r})=\left[\frac{2\left(\mu \omega^{\prime}\right)^{2 \eta+\frac{1}{2}} n_{r} !}{\Gamma\left(2 \eta+n_{r}+\frac{1}{2}\right)}\right]^{\frac{1}{2}} r^{2 \eta-1} \exp \left(-\frac{\mu \omega^{\prime} r^{2}}{2}\right) \times \\
\times F\left(-n_{r}, 2 \eta+\frac{1}{2} ; \mu \omega^{\prime} r^{2}\right) Y_{l, m}(\theta, \varphi) \\
E_{n, l}=-\frac{1}{8} a^{2} \omega^{2} \gamma+\frac{a \omega}{8} \sqrt{a^{2} \omega^{2} \gamma^{2}+16}
\end{gathered}
$$

where $a=4 n_{r}-2+\sqrt{(2 l+1)^{2}+8 \varpi g}$. The hypergeometric series $F\left(-n_{r}, 2 \eta+\frac{1}{2} ; \mu \omega^{\prime} r^{2}\right)$ can be replacing by $\frac{n_{r} !\left(2 \eta+\frac{3}{2}\right) !}{\left(n_{r}+2 \eta+\frac{3}{2}\right) !} L_{n_{r}}^{2 \eta+\frac{3}{2}}\left(\mu \omega^{\prime} r^{2}\right)$ to rewetting eignenfunctions as:

$$
\begin{aligned}
& \Psi_{n, l, m}(\stackrel{\leftrightarrow}{r})=\frac{n_{r} !\left(2 \eta+\frac{3}{2}\right) !}{\left(n_{r}+2 \eta+\frac{3}{2}\right) !}\left[\frac{2\left(\mu \omega^{\prime}\right)^{2 \eta+\frac{1}{2}} n_{r} !}{\Gamma\left(2 \eta+n_{r}+\frac{1}{2}\right)}\right]^{\frac{1}{2}} \times \\
& \times r^{2 \eta-1} \exp \left(-\frac{\mu \omega^{\prime} r^{2}}{2}\right) L_{n_{r}}^{2 \eta+\frac{3}{2}}\left(\mu \omega^{\prime} r^{2}\right) Y_{l, m}(\theta, \varphi)
\end{aligned}
$$

\section{BOOPP'S SHIFT METHOD FORMALISM}

In order to obtain the modified Schrödinger equation in noncommutative space and phase, we apply the following principal steps for energy dependent potential $V_{d p}\left(\hat{r}, E_{n, l}\right)[30-50]$ :

- We replace the ordinary Hamiltonian $\hat{H}\left(p_{i}, x_{i}\right)$ by noncommutative phase-space Hamiltonian $\hat{H}\left(\hat{p}_{i}, \hat{x}_{i}\right)$

- We replace the ordinary complex wave function $\Psi(\vec{r})$ by noncommutative complex wave function $\hat{\Psi}(\overrightarrow{\vec{r}})$;

- We replace the ordinary energy $E_{n, l}$ by new values $E_{n c d p}$ and the old product replace by star product (*).

Then, we can form the new Schrödinger equation in noncommutative space and phase as follows:

$$
\begin{aligned}
& \hat{H}\left(p_{i}, x_{i}\right) \Psi(\vec{r})=E_{n, l} \Psi(\vec{r}) \rightarrow \\
& \hat{H}\left(\hat{p}_{i}, \hat{x}_{i}\right) * \hat{\Psi}(\overrightarrow{\hat{r}})=E_{n c-d p} \hat{\Psi}(\overrightarrow{\hat{r}})
\end{aligned} \rightarrow
$$

In order to solve the above deformed equation, we apply the Boopp's shift method, which leads to reduce the above equation to simplest physical form:

$$
H_{n c-d p}\left(\hat{p}_{i}, \hat{x}_{i}\right) \psi(\vec{r})=E_{n c d p} \psi(\vec{r})
$$

The new modified Hamiltonian $H_{n c-d p}\left(\hat{p}_{i}, \hat{x}_{i}\right)$ ob- 
tained from ordinary Hamiltonian $\hat{H}\left(p_{i}, x_{i}\right)$ by applying the two translations $x_{i} \rightarrow \hat{x}_{i}$ and $p_{i} \rightarrow \hat{p}_{i}$ in eq. (3.2) as follows:

$$
H_{n c-d p}\left(\hat{p}_{i}, \hat{x}_{i}\right)=\frac{\hat{p}_{i}^{2}}{2 \mu}+V_{e p}(\hat{r})
$$

where the modified potential $V_{d p}\left(\hat{r}, E_{n, l}\right)$ is obtained from ordinary potential by:

$$
V_{d p}\left(\hat{r}, E_{n, l}\right)=\frac{1}{2} \mu \omega^{2} \hat{r}^{2}\left(1+\gamma E_{n, l}\right)+\frac{g}{\hat{r}^{2}}
$$

We have seen in our references [37-40], the two operators $\hat{r}^{2}$ and $\hat{p}^{2}$ in noncommutative three dimensional spaces and phases as follows:

$$
\hat{r}^{2}=r^{2}-\overrightarrow{\mathbf{L}} \vec{\Theta} \quad \text { and } \quad \hat{p}^{2}=p^{2}+\overrightarrow{\mathbf{L}} \overrightarrow{\boldsymbol{\theta}}
$$

Where $\mathbf{L} \Theta$ and $\overrightarrow{\mathbf{L}} \overrightarrow{\boldsymbol{\theta}}$ denotes to:

$$
\mathbf{L} \Theta \equiv L_{x} \Theta_{12}+L_{y} \Theta_{23}+L_{z} \Theta_{13}
$$

And

$$
\overrightarrow{\mathbf{L}} \overrightarrow{\boldsymbol{\theta}} \equiv L_{x} \bar{\theta}_{12}+L_{y} \bar{\theta}_{23}+L_{z} \bar{\theta}_{13}
$$

with $\Theta \equiv \frac{\theta}{2}$, which allow us to obtaining the second radial part in modified potential as:

$$
\frac{g}{\hat{r}^{2}}=\frac{g}{r^{2}}+\frac{g}{r^{4}} \overrightarrow{\mathbf{L}} \vec{\Theta}
$$

Therefore equations (19) and (18) in equation (17), we have:

$$
\begin{aligned}
& V_{d p}\left(\hat{r}, E_{n, l}\right)=\frac{1}{2} \mu \omega^{2} r^{2}\left(1+\gamma E_{n, l}\right)+ \\
& +\frac{g}{r^{2}}+V_{p e r t-d p}(r, \Theta, \bar{\theta})
\end{aligned}
$$

It's clearly that, the first 2 -terms in above equation represent the ordinary energy dependent potential, in commutative space, while the rest term is produced by the deformation of space and phase. The global perturbative potential operators $V_{p e r t-d p}(r, \Theta, \bar{\theta})$ for modified energy dependent potential in both (NC_3D: RSP) will be written as:

$$
V_{p e r t-d p}(r, \Theta, \bar{\theta})=\left(\frac{g}{r^{4}}-\frac{1}{2} \mu \omega^{2}\left(1+\gamma E_{n, l}\right)\right) \overrightarrow{\mathbf{L}} \vec{\Theta}+\frac{\overrightarrow{\mathbf{L}} \overrightarrow{\boldsymbol{\theta}}}{2 \mu}(23)
$$

\section{THE NONCOMMUTATIVE SPIN-ORBITAL HAMILTONIAN OPERATOR FOR MODIFIED ENERGY DEPENDENT POTENTIAL IN (NC_3D: RSP)}

Furthermore, to gives a physical interpretation to the eq. (23), we apply the same strategy, which we have applied in our previous work [30-44], we replace both
$\overrightarrow{\mathbf{L}} \vec{\Theta}$ and $\overrightarrow{\mathbf{L}} \overrightarrow{\boldsymbol{\theta}}$ by $2 \Theta \overleftrightarrow{S} \overleftrightarrow{L}$ and $2 \bar{\theta} \overleftrightarrow{S} \overleftrightarrow{L}$, respectively, to obtain the new forms of $H_{\text {pert-dp }}(r, \Theta, \bar{\theta})$ for modified energy dependent potential $V_{e p}(\hat{r})$ :

$$
\begin{aligned}
& H_{\text {pert-dp }}(r, \Theta, \bar{\theta})= \\
& =\left\{\Theta\left[\frac{g}{r^{4}}-\frac{1}{2} \mu \omega^{2}\left(1+\gamma E_{n, l}\right)\right]+\frac{\bar{\theta}}{2 \mu}\right\} \overrightarrow{\mathbf{L}} \vec{S}
\end{aligned}
$$

Here $\vec{S}$ denote the spin of quarkouniom systems $(c \bar{c}$ and $b \bar{b}$ ), The quantity $\overrightarrow{\mathbf{L}} \vec{S}$ is the scalar product of the orbital and intrinsic angular momentum operators, which can be replace by the equivalent operator $G^{2} \equiv \frac{1}{2}\left(\vec{J}^{2}-\vec{L}^{2}-\vec{S}^{2}\right)$ allow us to obtain the new physical form of eq. (24) as:

$$
\begin{aligned}
& H_{\text {pert-mt }}(r, \Theta, \bar{\theta})= \\
& =\frac{1}{2}\left\{\Theta\left[\frac{g}{r^{4}}-\frac{1}{2} \mu \omega^{2}\left(1+\gamma E_{n, l}\right)\right]+\frac{\bar{\theta}}{2 \mu}\right\}\left(\vec{J}^{2}-\vec{L}^{2}-\vec{S}^{2}\right)
\end{aligned}
$$

Where $\vec{J}$ is the sum of $\vec{L}$ and $\vec{S}$, it is well known, that the eigenvalues of the total operator can be obtains from the interval $|l-s| \leq j \leq|l+s|$, which allow us to obtaining the eigenvalues of the operator $G^{2}$ as $k(j, l, s) \equiv j(j+1)+l(l+1)-s(s+1)$.

\subsection{The Exact Spin-orbital Spectrum for Spin- orbital Hamiltonian in (NC_ 3D: RSP)}

The main goal of this section is to calculate the modifications to the energy levels $E_{\text {ep }}$ at first order of two parameters $\Theta$ and $\bar{\theta}$ of quarkouniom systems $(c \bar{c}$ and $b \bar{b})$ described by the modified Schrödinger equation, by applying the standard perturbation theory to obtain:

$$
\begin{aligned}
& E_{\text {ep }}=\frac{\left(\mu \omega^{\prime}\right)^{2 \eta+\frac{1}{2}}\left(n_{r} !\right)^{2}\left(2 \eta+\frac{3}{2}\right) ! k(j, l, s)}{\left(n_{r}+2 \eta+\frac{3}{2}\right) ! \Gamma\left(2 \eta+n_{r}+\frac{1}{2}\right)} \times \\
& \times \int_{0}^{+\infty} r^{4 \eta} \exp \left(-\mu \omega^{\prime} r^{2}\right)\left[L_{n_{r}}^{2 \eta+\frac{3}{2}}\left(\mu \omega^{\prime} r^{2}\right)\right]^{2} \times \\
& \times\left\{\Theta\left[\frac{g}{r^{4}}-\frac{1}{2} \mu \omega^{2}\left(1+\gamma E_{n, l}\right)\right]+\frac{\bar{\theta}}{2 \mu}\right\} d r
\end{aligned}
$$

A direct simplification gives:

$$
\begin{aligned}
& E_{\mathrm{ep}}=\frac{\left(\mu \omega^{\prime}\right)^{2 \eta+\frac{1}{2}}\left(n_{r} !\right)^{2}\left(2 \eta+\frac{3}{2}\right) ! k(j, l, s)}{\left(n_{r}+2 \eta+\frac{3}{2}\right) ! \Gamma\left(2 \eta+n_{r}+\frac{1}{2}\right)} \times \\
& \times\left\{\Theta \sum_{i=1}^{2} T_{i}+\frac{\bar{\theta}}{2 \mu} T_{3}\right\}
\end{aligned}
$$


Where, the three terms $T_{i}(i=\overline{1,3})$ are given by:

$$
\begin{aligned}
& T_{1}=g \int_{0}^{+\infty} r^{4 \eta-4} \exp \left(-\mu \omega^{\prime} r^{2}\right)\left[L_{n_{r}}^{2 \eta+\frac{3}{2}}\left(\mu \omega^{\prime} r^{2}\right)\right]^{2} d r, \\
& T_{2}=-\frac{1}{2} \mu \omega^{2}\left(1+\gamma E_{n, l}\right) \int_{0}^{+\infty} r^{4 \eta} \exp \left(-\mu \omega^{\prime} r^{2}\right) \times \\
& \times\left[L_{n_{r}}^{2 \eta+\frac{3}{2}}\left(\mu \omega^{\prime} r^{2}\right)\right]^{2} d r, \\
& T_{3}=\frac{1}{2 \mu} \int_{0}^{+\infty} r^{4 \eta} \exp \left(-\mu \omega^{\prime} r^{2}\right)\left[L_{n_{r}}^{2 \eta+\frac{3}{2}}\left(\mu \omega^{\prime} r^{2}\right)\right]^{2} d r
\end{aligned}
$$

Now, we set $\mu \omega^{\prime} r^{2}=\delta t$ and then the above 3 -terms are reducing to the following form:

$$
\begin{aligned}
& T_{1}=\frac{g}{2} \int_{0}^{+\infty} t^{\left(2 \eta-\frac{1}{2}\right)-1} \exp \left(-\mu \omega^{\prime} t\right)\left[L_{n_{r}}^{2 \eta+\frac{3}{2}}\left(\mu \omega^{\prime} t\right)\right]^{2} d t, \\
& T_{2}=-\frac{1}{4} \mu \omega^{2}\left(1+\gamma E_{n, l}\right) \int_{0}^{+\infty} t^{\left(2 \eta+\frac{1}{2}\right)^{-1}} \times \\
& \times \exp \left(-\mu \omega^{\prime} t\right)\left[L_{n_{r}}^{2 \eta+\frac{3}{2}}\left(\mu \omega^{\prime} t\right)\right]^{2} d t, \\
& T_{3}=\frac{1}{4 \mu} \int_{0}^{+\infty} t^{\left.2 \eta+\frac{1}{2}\right)^{-1}} \exp \left(-\mu \omega^{\prime} t\right)\left[L_{n_{r}}^{2 \eta+\frac{3}{2}}\left(\mu \omega^{\prime} t\right)\right]^{2} d t
\end{aligned}
$$

Applying the following special integration [52, 53]:

$$
\begin{aligned}
& \int_{0}^{+\infty} t^{\alpha-1 .} \exp (-\delta t) \Gamma_{m}^{\lambda}(\delta t) \Gamma_{n}^{\beta}(\delta t) d t= \\
& =\frac{\delta^{-\alpha} \Gamma(n-\alpha+\beta+1) \Gamma(m+\lambda+1)}{m ! n ! \Gamma(1-\alpha+\beta) \Gamma(1+\lambda)} \times \\
& \times{ }_{3} F_{2}(-m, \alpha, \alpha-\beta ;-n+\alpha, \lambda+1 ; 1)
\end{aligned}
$$

Where ${ }_{3} F_{2}(-m, \alpha, \alpha-\beta ;-n+\alpha, \lambda+1 ; 1)$ denote to the hypergeometric function, it's obtained from

$$
{ }_{p} F_{q}\left(\alpha_{1}, \ldots, \alpha_{p}, \beta_{1}, \ldots ., \beta_{q}, z\right) \text { For: } p=3 \text { and } q=2,
$$

This is a generalized hypergeometric series. After straightforward calculations, we can obtain the explicitly results:

$$
\begin{aligned}
& T_{1}=\frac{g}{2} \frac{\left(\mu \omega^{\prime}\right)^{-\left(2 \eta-\frac{1}{2}\right)} \Gamma\left(n_{r}+3\right) \Gamma\left(n_{r}+2 \eta+\frac{5}{2}\right)}{\left(n_{r} !\right)^{2} \Gamma(3) \Gamma\left(2 \eta+\frac{5}{2}\right)} \\
& \ldots ._{3} F_{2}\left(-n_{r}, 2 \eta-\frac{1}{2},-2 ;-n_{r}+2 \eta-\frac{1}{2}, 2 \eta+\frac{5}{2} ; 1\right) \\
& T_{2}=-\frac{1}{4} \mu \omega^{2}\left(1+\gamma E_{n, l}\right) \frac{\left(\mu \omega^{\prime}\right)^{-\left(2 \eta+\frac{1}{2}\right)} \Gamma\left(n_{r}-2\right) \Gamma\left(n_{r}+2 \eta+\frac{5}{2}\right)}{\left(n_{r} !\right)^{2} \Gamma(2) \Gamma\left(2 \eta+\frac{5}{2}\right)} \\
& \ldots \ldots{ }_{3} F_{2}\left(-n_{r}, 2 \eta+\frac{1}{2},-1 ;-n_{r}+2 \eta+\frac{1}{2}, 2 \eta+\frac{5}{2} ; 1\right)
\end{aligned}
$$

$$
\begin{aligned}
& T_{3}=\frac{1}{4 \mu} \frac{\left(\mu \omega^{\prime}\right)^{-\left(2 \eta+\frac{1}{2}\right)} \Gamma\left(n_{r}-2\right) \Gamma\left(n_{r}+2 \eta+\frac{5}{2}\right)}{\left(n_{r} !\right)^{2} \Gamma(2) \Gamma\left(2 \eta+\frac{5}{2}\right)} \\
& \ldots \ldots \ldots \ldots \ldots{ }_{3} F_{2}\left(-n_{r}, 2 \eta+\frac{1}{2},-1 ;-n_{r}+2 \eta+\frac{1}{2}, 2 \eta+\frac{5}{2} ; 1\right)
\end{aligned}
$$

Inserting equation (31) in the modified energy levels equation (27) gives:

$$
\begin{aligned}
& E_{\text {ep }}=\frac{\left(\mu \omega^{\prime}\right)^{2 \eta+\frac{1}{2}}\left(2 \eta+\frac{3}{2}\right) ! \Gamma\left(n_{r}+2 \eta+\frac{5}{2}\right) k(j, l, s)}{\left(n_{r}+2 \eta+\frac{3}{2}\right) ! \Gamma\left(2 \eta+n_{r}+\frac{1}{2}\right) \Gamma\left(2 \eta+\frac{5}{2}\right)} \times \\
& \times\left\{\theta T_{n c-s}+\frac{\bar{\theta}}{2 \mu} T_{n c-p}\right\}
\end{aligned}
$$

Where $T_{n c-s}$ and $T_{n c-p}$ are given by:

$$
\begin{aligned}
& T_{n c-s} \equiv \frac{g}{2} \frac{\left(\mu \omega^{\prime}\right)^{-\left(2 \eta-\frac{1}{2}\right)} \Gamma\left(n_{r}+3\right)}{\Gamma(3)} \times \\
& \times_{3} F_{2}\left(-n_{r}, 2 \eta-\frac{1}{2},-2 ;-n_{r}+2 \eta-\frac{1}{2}, 2 \eta+\frac{5}{2} ; 1\right)- \\
& -\frac{1}{4} \mu \omega^{2}\left(1+\gamma E_{n, l}\right) \frac{\left(\mu \omega^{\prime}\right)^{-\left(2 \eta+\frac{1}{2}\right)} \Gamma\left(n_{r}-2\right)}{\Gamma(2)} \times \\
& \times_{3} F_{2}\left(-n_{r}, 2 \eta+\frac{1}{2},-1 ;-n_{r}+2 \eta+\frac{1}{2}, 2 \eta+\frac{5}{2} ; 1\right)
\end{aligned}
$$

And

$$
\begin{aligned}
& T_{n c-p} \equiv \frac{1}{4 \mu} \frac{\left(\mu \omega^{\prime}\right)^{-\left(2 \eta+\frac{1}{2}\right)} \Gamma\left(n_{r}-2\right)}{\Gamma(2)} \times \\
& \times_{3} F_{2}\left(-n_{r}, 2 \eta+\frac{1}{2},-1 ;-n_{r}+2 \eta+\frac{1}{2}, 2 \eta+\frac{5}{2} ; 1\right)
\end{aligned}
$$

\subsection{The Exact Magnetic Spectrum for Spin- orbital Hamiltonian in (NC_ 3D: RSP)}

Furthermore, we can found another automatically symmetry for modified the energy dependent potential related to the influence of an external uniform magnetic field, it's deduced by the following two simultaneously replacements:

$$
\left\{\begin{array}{l}
\Theta \rightarrow \xi B \\
\bar{\theta} \rightarrow \bar{\tau} B
\end{array}\right.
$$

Here $\xi$ and $\bar{\tau}$ are infinitesimal real proportional's constants and to simplify our calculations, we choose the magnetic field $\overleftrightarrow{B}=B \vec{k}$ and then we can make the following translation:

$$
\begin{aligned}
& \frac{1}{2}\left\{\Theta\left(\frac{g}{r^{4}}-\frac{1}{2} \mu \omega^{2}\left(1+\gamma E_{n, l}\right)\right)+\frac{\bar{\theta}}{2 \mu}\right\} \vec{B} \vec{L} \rightarrow \\
& \left(\xi\left(\frac{g}{r^{4}}-\frac{1}{2} \mu \omega^{2}\left(1+\gamma E_{n, l}\right)\right)+\frac{\bar{\tau}}{2 \mu}\right) B L_{z}
\end{aligned}
$$


Which allow us to introduce the modified new magnetic Hamiltonian operator $\hat{H}_{m-e d}$ in (NC_3D: RSP) as:

$$
\hat{H}_{m-e d}=\left(\xi\left(\frac{g}{r^{4}}-\frac{1}{2} \mu \omega^{2}\left(1+\gamma E_{n, l}\right)\right)+\frac{\bar{\tau}}{2 \mu}\right)(\vec{B} \vec{J}-\overleftrightarrow{S} \overleftrightarrow{B})
$$

It is clearly, that the above operator present the magnetic effect in (NC_3D: RSP), including ordinary magnetic effect $(-\overleftrightarrow{S} \overleftrightarrow{B})$. To obtain the exact noncommutative magnetic modifications of energy $E_{\text {mag-ed }}$ for energy dependent potential, we replace:

$$
\left\{\begin{array}{l}
k(j, l, s) \rightarrow m \\
\Theta \rightarrow \xi \\
\bar{\theta} \rightarrow \bar{\tau}
\end{array}\right.
$$

Substitution of above replacement into equation (32) allow us to obtains the modification of the energy levels corresponding the magnetic effect

$$
\begin{aligned}
& E_{\text {mag-ed }}=\frac{\left(\mu \omega^{\prime}\right)^{2 \eta+\frac{1}{2}}\left(2 \eta+\frac{3}{2}\right) ! \Gamma\left(n_{r}+2 \eta+\frac{5}{2}\right) B}{\left(n_{r}+2 \eta+\frac{3}{2}\right) ! \Gamma\left(2 \eta+n_{r}+\frac{1}{2}\right) \Gamma\left(2 \eta+\frac{5}{2}\right)} \times \\
& \times m\left\{\xi T_{n c-s}+\frac{\bar{\tau}}{2 \mu} T_{n c-p}\right\}
\end{aligned}
$$

with $-l \leq m \leq+l$.

\section{THE EXACT GLOBAL SPECTRUM FOR MOD- IFIED MIE-TYPE POTENTIALS IN NC_3D: RSP}

We now summarize obtained global energy levels $E_{\text {nc ed }}$ for the modified energy dependent potential of quarkouniom systems ( $\bar{c}$ and $b \bar{b}$ ) in (NC_3D: RSP) as provided in subsections (4.1) and (4.2), according to three equations (13), (32) and (39) the explicit form for $E_{\text {nc ed }}$ is then:

$$
\begin{aligned}
& E_{\text {nc ed }}=-\frac{1}{8} a^{2} \omega^{2} \gamma+ \\
& \frac{\left(\mu \omega^{\prime}\right)^{2 \eta+\frac{1}{2}}\left(2 \eta+\frac{3}{2}\right) ! \Gamma\left(n_{r}+2 \eta+\frac{5}{2}\right) k(j, l, s)}{\left(n_{r}+2 \eta+\frac{3}{2}\right) ! \Gamma\left(2 \eta+n_{r}+\frac{1}{2}\right) \Gamma\left(2 \eta+\frac{5}{2}\right)} \times \\
& \times\left\{\theta T_{n c-s}+\frac{\bar{\theta}}{2 \mu} T_{n c-p}\right\}+\ldots
\end{aligned}
$$

$$
\begin{aligned}
& \ldots+\frac{\left(\mu \omega^{\prime}\right)^{2 \eta+\frac{1}{2}}\left(2 \eta+\frac{3}{2}\right) ! \Gamma\left(n_{r}+2 \eta+\frac{5}{2}\right) B}{\left(n_{r}+2 \eta+\frac{3}{2}\right) ! \Gamma\left(2 \eta+n_{r}+\frac{1}{2}\right) \Gamma\left(2 \eta+\frac{5}{2}\right)} \times \\
& \times m\left\{\xi T_{n c-s}+\frac{\bar{\tau}}{2 \mu} T_{n c-p}\right\}
\end{aligned}
$$

It is well known that the atomic quantum number $m$ and $j$ can be takes $(2 l+1)$ values and $\underbrace{j=|l-s|,|l-s|+1, \ldots, \ldots j=|l+s| \text { and } j=|l+s|}_{\text {N-possible values for } \mathrm{j}}$ values, respectively, thus every old state in usually three dimensional space of energy dependent potential will be $N(2 l+1)$ sub-states for modified energy dependent potential $V_{n c-e d}(r)$ in (NC_3D: RSP). Moreover, from the previous obtained results, presented in eq. (25) and eq. (37), in addition to the usually Hamiltonian operator in eq. (5), we can deduce the global noncommutative Hamiltonian matrix $\hat{H}_{n c-e d}$ in both (NC_3D: RSP) for studied potential:

$$
\begin{aligned}
& \hat{H}_{n c-e d}=-\frac{\Delta}{2 \mu}+\frac{1}{2} \mu \omega^{2} r^{2}\left(1+\gamma E_{n, l}\right)+\frac{g}{r^{2}}+ \\
& +\left\{\Theta\left[\frac{g}{r^{4}}-\frac{1}{2} \mu \omega^{2}\left(1+\gamma E_{n, l}\right)\right]+\frac{\bar{\theta}}{2 \mu}\right\} \overrightarrow{\mathbf{L}} \vec{S}+ \\
& +\left(\xi\left(\frac{g}{r^{4}}-\frac{1}{2} \mu \omega^{2}\left(1+\gamma E_{n, l}\right)\right)+\frac{\bar{\tau}}{2 \mu}\right)(\vec{B} \vec{J}-\vec{S} \vec{B})
\end{aligned}
$$

It's clearly, that the obtained eigenvalues of energies are reels and then the noncommutative diagonal Hamiltonian $\hat{H}_{n c-e d}$ is Hermitian.

\section{CONCLUSIONS}

In this work, the Schrödinger equation in noncommutative phase-space three dimension has been solved exactly for the bound states corresponding to energy dependent potential plus three new terms produced by the new geometry of space and phase. Moreover we have formed a corresponding noncommutative modified operator Hamiltonian for studied potential.

\section{ACKNOWLEDGMENTS}

This work was supported with search laboratory of Physics and Material Chemistry in University of M'sila, Algeria. 


\section{REFERENCES}

1. M.S. Child M.S. Shi-Hai Dong, X.G. Wang, J. Phys. A 33 No 32, 5653 (2000).

2. S.K. Bose, Il Nuovo Cimento B 109 No 11, 1217 (1994).

3. L. Buragohain L, S.A.S. Ahmed, Lat. Am. J. Phys. Educ. 4 No 1, 79 (2010).

4. S.A.S. Ahmed, B.C. Borah, D. Sarma, Eur. Phys. J. D 17 No 1, 5 (2001)

5. S.M. Ikhdair, R. Sever, CEJP 5 No 4, 516 (2007).

6. M.M. Nieto, Am. J. Phys. 47, 1067 (1979).

7. S.M. Ikhdair, R. Sever, J. Mol. Struct. Theochem. 806, 155 (2007).

8. S.A.S. Ahmed, L. Buragohain, Phys. Scr. 80 No 2, 4 (2009).

9. S.K. Bose, N. Gupta, Nuovo Cimento B 113, 299 (1998).

10. G.P. Flesses, A. Watt, J. Phys. A: Math. Gen. 14 No 9, L315 (1981).

11. S.M. Ikhdair, R. Sever, Ann. Phys. (Leipzig) 16 No 3, 218 (2007).

12. Shi-Hai Dong, Phys. Scr. 64 No 4, 273 (2001).

13. Shi-Hai Dong, Z.Q. Ma, J. Phys. A 31 No 49, 9855 (1998).

14. Shi-Hai Dong, Int. J. Theor. Phys. 40 No 2, 559 (2001).

15. A.A. Rajabi, M. Hamzavi, J. Theor. Appl. Phys. 7, 17 (2013).

16. S.M. Ikhdair, R. Sever, Adv. High Energy Phys. 2013, ID 562959 (2013).

17. Shi-Hai Dong, G.H. San, Found. Phys. Lett. 16 No 4, 357 (2003).

18. L. Buragohain, S.A.S. Ahmed, Lat. Am. J. Phys. Educ. 4 No 1, 79 (2010).

19. S.M. Ikhdair, J. Mod. Phys. 3 No 2, 170 (2012).

20. H. Hassanabadi, E. Maghsoodi, R. Oudi, S. Zarrinkamar, H. Rahimov, Eur. Phys. J. Plus. 127, 120 (2012).

21. H. Hassanabadi, M. Hamzavi, S. Zarrinkamar, A.A. Rajabi, Int. J. Phys. Sci. 6 No 3, 583 (2011).

22. Shi-Hai Dong, Z.Q. Ma, G. Esposito, Found. Phys. Lett. 12 No 5, 465 (1999).

23. Gupta Pramila, Mehrotra Indira, J. Modern Phys. 3, 1530 (2012).

24. Kayode John Oyewumi, Ezekiel Adeniyi Bangudu, The Arabian J. Sci. Eng. 28 No 2A, 173 (2003).

25. Shi-Hai Dong, Guo-Hua Sun, Phys. Scr. 70, 94 (2004).

26. D.T. Jacobus, Department of Physics, Stellenbosch University, South Africa (2010).
27. Z.H. Yang, C.Y. Long, S.J. Qin, Z.W. Long, Int. J. Theor. Phys. 49 No 3, 644 (2010).

28. Y. Yuan, L. Kang, W. Jian-Hua, C. Chi-Yi, Chin. Phys. C 34 No 5, 543 (2010)

29. B. Mirza, R. Narimani, S. Zare, Commun. Theor. Phys. 55, 405 (2011)

30. A. Maireche, Life Sci. J. 11 No 6, 353 (2014).

31. A. Maireche, Afr. Rev. Phys. 9, 479 (2014).

32. A. Maireche, J. Nano- Electron. Phys. 7 No 2, 02003 (2015).

33. A. Maireche, Afr. Rev. Phys. 9, 185 (2014).

34. A. Maireche, Afr. Rev. Phys. 10:0014, 97 (2015).

35. A. Maireche, Int. Lett. Chem., Phys. Astronomy 56, 1 (2015).

36. A. Maireche, Int. Lett. Chem., Phys. Astronomy 60, 11 (2015).

37. A. Maireche, Afr. Rev. Phys. 10:0025, 177 (2015).

38. A. Maireche, Afr. Rev. Phys. 10:0046, 373 (2015).

39. A. Maireche, Int. Lett. Chem., Phys. Astronomy, 58, 164 (2015).

40. A. Maireche, Med. J. Model. Simul. 04, 060 (2015).

41. A. Maireche, Int. Lett. Chem., Phys. Astronomy, 61, 38 (2015).

42. A. Maireche, J. Nano- Electron. Phys. 7 No 4, 04021 (2015).

43. A. Maireche, J. Nano- Electron. Phys. 7 No 3, 03047 (2015).

44. A. Maireche, Lat. Am. J. Phys. Educ. 9 No 1, 1301 (2015).

45. A. Maireche, Nano World J. 1 No 4, 120 (2015).

46. S. Cai, T. Jing, G. Guo, R. Zhang, Int. J. Theoret. Phys. 49 No 8, 1699 (2010).

47. J. Lee, J. Korean Phys. Soc. 47 No 4, 571 (2005).

48. A. Jahan, Braz. J. Phys. 38, 144 (2008).

49. A.E.F. Djemei, H. Smail, Commun. Theor. Phys. 41, 837 (2004).

50. A. Smailagic, E. Spallucci, Phys. Rev. D 65, 107701 (2002).

51. H. Snyder, Phys. Rev. 71, 38 (1947).

52. Altug Arda, Ramazan Server, Commun. Theor. Phys. 58 No 1, 27 (2012).

53. M. Abramowitz, I.A. Stegun, Handbook of Mathematical Functions with Formulas, Graphs, and Mathematical Tables (Dover Publications: 1965). 